\title{
One Small Step for Empagliflozin, One Giant Leap for Diabetology
}

Sanjay Kalra

To view enhanced content go to www.diabetestherapy-open.com

Received: October 5, 2015 / Published online: November 4, 2015

(c) The Author(s) 2015. This article is published with open access at Springerlink.com

\section{ABSTRACT}

This article discusses the recently published EMPA-REG OUTCOME trial, which assessed cardiovascular outcomes with empagliflozin therapy in persons with type 2 diabetes mellitus and coexisting cardiovascular disease. The article describes the background and challenges of modern cardiovascular outcome trials, points out the strengths of the EMPA-REG OUTCOME study, and places the results in perspective. It highlights the significant impact that these results will have on cardiovascular preventive pharmacotherapy, and on future drug development in diabetes. At the same time, it reminds readers of the limitations of the results, and lists the questions raised by, or left unanswered by, the trial.

Keywords: Canagliflozin; Cardiovascular outcomes; Dapagliflozin; Empagliflozin; Sodium-glucose co-transporter 2 (SGLT2) inhibitors; Type 2 diabetes mellitus

S. Kalra $(\bowtie)$

Department of Endocrinology, Bharti Hospital, Karnal, India

e-mail: brideknl@gmail.com

\section{INTRODUCTION}

The EMPA-REG OUTCOME trial (ClinicalTrials.gov identifier, NCT01131676) recently announced the effects of empagliflozin on the cardiovascular outcomes (CVO) and mortality in persons with type 2 diabetes mellitus (T2DM) [1]. In this seminal CVO trial (CVOT), 7020 patients with T2DM with coexisting cardiovascular disease (CVD; myocardial infarction [MI], stroke, or peripheral arterial disease) were randomized to either $10 \mathrm{mg}$ empagliflozin, $25 \mathrm{mg}$ empagliflozin, or placebo, over and above standard of care. The primary composite outcome was a total of three endpoints (death from CV causes, non-fatal MI, and non-fatal stroke), while the key secondary endpoint included a fourth endpointhospitalization for unstable angina-in addition to the composite primary endpoint [1].

The results of this study revealed a statistically significant reduction in the primary endpoint with empagliflozin use (10.5\% in the empagliflozin group vs. $12.1 \%$ in the placebo group; hazard ratio 0.86 ; relative risk reduction (RRR) 14\%, 95\% confidence interval $0.74-0.99 ; P=0.04)$. Similarly, a RRR 
reduction is noted in death from $\mathrm{CV}$ causes (RRR 38\%; 3.7\% vs. 5.9\%), hospitalization for heart failure (RRR 35\%; $2.7 \%$ vs. $4.1 \%$ ), and all-cause death (RRR 32\%; $5.7 \%$ vs. $8.3 \%$ ). However, the difference in rates of non-fatal MI and non-fatal stroke did not reach statistical significance.

This article is based on previously conducted studies and does not involve any new studies of human or animal subjects performed by the author.

\section{EARLIER CARDIOVASCULAR OUTCOME TRIALS}

Earlier trials have also reported $\mathrm{CVO}$ with various glucose-lowering medications. The University Group Diabetes Program (UGDP) study, published nearly half a century ago, highlighted the negative effects of tolbutamide on CV health [2]. The United Kingdom Prospective Diabetes Study (UKPDS), a large study with multiple arms, unearthed the beneficial impact of metformin in contrast to other drugs such as chlorpropamide in improving $\mathrm{CVO}$ in T2DM [3]. The STOP-NIDDM trial, breaking new ground, suggested CVO improvement when acarbose was administered to persons with impaired glucose tolerance [4]. Similarly, the PROActive trial on pioglitazone reported a reduction in the composite of all-cause mortality, non-fatal MI, and stroke in patients with T2DM who have a high risk of macrovascular events [5]. However, all of these studies were not designed as a CVOT, and their primary aim was to assess glucose-lowering efficacy rather than improvement in $\mathrm{CV}$ health.

Advances in the understanding of the interlink between diabetes and $\mathrm{CV}$ disease (CVD), an appreciation of the need to improve
$\mathrm{CVO}$ in diabetes care, and the realization that effective glucose-lowering drugs could end up worsening CVO (e.g., muraglitazar [6]), have led to a mandatory requirement for CVOTs in anti-diabetic drugs pending regulatory approval.

It then became imperative for all newly developed glucose-lowering molecules to undergo CV safety analysis by performing a CVOT. The first drug to report such an analysis, after institution of new regulatory requirements, was quick-release bromocriptine, which demonstrated CV safety in a trial which recruited 3070 subjects for a mean follow up of 52 weeks [7]. Since then, various data on dipeptidyl-peptidase 4 inhibitors [8-10] and insulins [11] have been published from large-scale CVOTs which have suggested their CV safety.

While these CVOTs follow similar trial designs and protocols, subtle variations are present [8-11]. Differences in inclusion/ exclusion criteria, the duration of follow up, and the choice of primary/secondary endpoints can be easily discerned. Though debate around the validity of various trial designs is never ending, there is broad consensus that modern CVOTs are well designed and have good reliability. All recent CVOTs have been designed to demonstrate $\mathrm{CV}$ safety (non-inferiority), rather than superiority, as requested by regulators. This fact should be understood for rational interpretation of their results.

\section{IMPROVEMENT IN CARDIOVASCULAR CARE}

Over the past few decades the standard of care for $\mathrm{CV}$ prevention has improved markedly across the globe. Use of medical interventions such as 
aspirin, statins, angiotensin-converting-enzyme inhibitors (ACEi), angiotensin receptor blockers (ARBs), and beta blockers has helped to enhance CVO, especially in persons with T2DM. Though widespread use of these standard of care drugs allow easier demonstration of CV safety, their use makes it difficult for a new add-on therapy to demonstrate improvement in CVO.

\section{EMPAGLIFLOZIN: BETTER BENEFIT}

This is exactly what empagliflozin has achieved. In a high-risk cohort of subjects, with over $75 \%$ usage of statins, over $80 \%$ use of ACEi/ARBs, and over $60 \%$ beta-blocker therapy, administration of empagliflozin was able to demonstrate added benefit in terms of CVO [1]. This speaks for the strength of the molecule being studied, as well as the robust quality of trial methodology followed by the authors of the study. Inclusion of a wide variety of high-risk subjects, from 42 different nationalities, enhances the global applicability of these results.

The benefits of empagliflozin were noted early on in the EMPA-REG OUTCOME trial, and continued throughout the study [1]. This finding makes empagliflozin stand apart from other CVD preventive drugs, such as statins and ramipril, which demonstrated a CV benefit after a longer duration of therapy. Whether this means that empagliflozin is an effective drug for secondary prevention of CVD, that is, the prevention of progression of CVD to death is open to debate. Detractors point to the lack of statistical significance noted with respect to occurrence of non-fatal MI and stroke. However, this finding may be thought to reinforce the "preventive power" of empagliflozin: It may even be used in tertiary CVD prevention, as it helps improve outcomes in persons with T2DM who experience an MI or stroke, ensuring that they survive the acute illness. Thus, empagliflozin may be useful for tertiary prevention, rather than secondary prevention, of CVD. The number needed to treat, (for all-cause mortality), which was 39 for empagliflozin over a period of 3 years, is much lower than that reported for other drugs, including ramipril and simvastatin $[12,13]$.

The highly significant benefit of empagliflozin $(P=0.002)$ in preventing hospitalization for heart failure raises hope for its use in this clinical situation [1]. This finding is especially welcome considering the controversy related to the increased risk of heart failure hospitalization with other glucose-lowering therapies [14]. Empagliflozin, therefore, gets evidence-backed justification for use not only as a glucose-lowering therapy, but also raises hope for its potential as adjunctive therapy for CVD prevention (Table 1).

\section{EMPAGLIFLOZIN: EXPANDING BOUNDARIES}

The EMPA-REG OUTCOME results should be carefully interpreted to assess their impact on diabetes care and outcomes. While the findings for different subsets of patients need to be studied separately, the EMPA-REG OUTCOME study also throws up a few interesting questions (Table 1). Will these beneficial results be relevant to persons with T2DM and low CV risk, or to persons with type 1 diabetes mellitus? Whether the findings of empagliflozin can be extrapolated to other sodium-glucose co-transporter 2 inhibitors (SGLT2i) are open to discussion. CVOTs are underway for both canagliflozin and dapagliflozin, and their results will decide whether the $\mathrm{CV}$ benefits of empagliflozin are a class effect or not [15]. 
Table 1 Impact of EMPA-REG OUTCOME study [1]

Strengths

Robust methodology/clinical design

Multinational coverage ( 42 countries)

Applicability across age, gender, CV phenotype

Clinically relevant inclusion/exclusion criteria

Clear-cut answers from straightforward statistical analysis

Positive answers

Empagliflozin is safe in high-risk CV patients with T2DM

Empagliflozin improves $\mathrm{CV}$ outcomes in high-risk $\mathrm{CV}$ patients with T2DM

Empagliflozin can be used safely in combination with other vascular-tropic drugs over an extended period of time

Empagliflozin can be used for prevention of CVD

Empagliflozin does not increase the risk of diabetic ketoacidosis or bone fractures

Unanswered questions

Is the beneficial effect of empagliflozin a class effect, or a property unique to this molecule?

What are the mechanisms that account for the potential of empagliflozin to reduce $\mathrm{CV}$ outcomes?

Is the beneficial $\mathrm{CV}$ effect of empagliflozin relevant to patients with T2DM and low CV risk, and to patients with type 1 diabetes mellitus?

Can empagliflozin be prescribed in acute coronary settings?

Can empagliflozin be considered an adjuvant preventive therapy for CVD?

$C V$ cardiovascular, $C V D$ cardiovascular disease, T2DM type 2 diabetes mellitus

It is also uncertain if empagliflozin can be initiated, or continue to be used, in acute coronary settings, including unstable angina, MI, and heart failure. However, while translating CVOT evidence to practice one should not lose sight of good clinical sense. SGLT2i biology and pharmacology should be understood in detail before prescribing SGLT2i [16]. This therapy should be accompanied by appropriate medication counseling and should not be prescribed to persons at risk of ketoacidosis or recurrent genital infections [17].

\section{SUMMARY}

While the EMPA-REG OUTCOME study findings should be interpreted carefully to assess their impact on diabetes care and outcomes, the results go far beyond proving the superiority of empagliflozin in improving CVO [1]. The seminal importance of these findings will ensure that this trial acts as a milestone in the fields of diabetology and CV medicine.

The EMPA-REG OUTCOME study raises the bar for future glucose-lowering drugs. It will not now suffice to demonstrate $\mathrm{CV}$ neutrality or $\mathrm{CV}$ safety. Rather, unequivocal evidence of $\mathrm{CV}$ benefits will be required. Such a development will help improve not only CVO, but also diabetes outcomes overall. The EMPA-REG OUTCOME study may be just a small step for empagliflozin, but is a giant leap for diabetes care.

\section{ACKNOWLEDGMENTS}

No funding or sponsorship was received for publication of this article. The named author meets the International Committee of Medical Journal Editors (ICMJE) criteria for authorship for this manuscript, takes responsibility for the integrity of the work as a whole, and has given final approval for the version to be published. 
Disclosures. Sanjay Kalra has received honoraria/speaker's fees from Boehringer Ingelheim, AstraZeneca, and Janssen.

Compliance with ethics guidelines. This article is based on previously conducted studies and does not involve any new studies of human or animal subjects performed by the author.

Open Access. This article is distributed under the terms of the Creative Commons Attribution-NonCommercial 4.0 International License (http://creativecommons.org/licenses/ by-nc/4.0/), which permits any noncommercial use, distribution, and reproduction in any medium, provided you give appropriate credit to the original author(s) and the source, provide a link to the Creative Commons license, and indicate if changes were made.

\section{REFERENCES}

1. Zinman B, Wanner C, Lachin JM. Glucose-lowering agent shows $\mathrm{CV}$ benefit in outcome trial, for the first time. N Engl J Med. 2015. doi:10.1056/ NEJMoa1504720. (Epub ahead of print).

2. Salsburg DS. The UGDP study. JAMA. 1971;218(11):1704-5.

3. UK Prospective Diabetes Study (UKPDS) Group. Effect of intensive blood-glucose control with metformin on complications in overweight patients with type 2 diabetes (UKPDS 34). Lancet. 1998;352(9131):854-65.

4. Chiasson JL, Josse RG, Gomis R, et al. Acarbose treatment and the risk of cardiovascular disease and hypertension in patients with impaired glucose tolerance: the STOP-NIDDM trial. JAMA. 2003;290(4):486-94.

5. Nissen SE, Wolski K, Topol EJ. Effect of muraglitazar on death and major adverse cardiovascular events in patients with type 2 diabetes mellitus. JAMA. 2005;294(20):2581-6.

6. Dormandy JA, Charbonnel B, Eckland DJ, et al. Secondary prevention of macrovascular events in patients with type 2 diabetes in the PROactive Study (PROspective pioglitAzone Clinical Trial In macroVascular Events): a randomised controlled trial. Lancet. 2005;366(9493):1279-89.

7. Gaziano JM, Cincotta AH, O'Connor CM, et al. Randomized clinical trial of quick-release bromocriptine among patients with type 2 diabetes on overall safety and cardiovascular outcomes. Diabetes Care. 2010;33(7):1503-8.

8. White WB, Cannon CP, Heller SR, et al. Alogliptin after acute coronary syndrome in patients with type 2 diabetes. N Engl J Med. 2013;369:1327-35.

9. Scirica BM, Bhatt DL, Braunwald E, et al. Saxagliptin and cardiovascular outcomes in patients with type 2 diabetes mellitus. N Engl J Med. 2013;369:1317-26.

10. Green JB, Bethel MA, Armstrong PW, et al. Effect of sitagliptin on cardiovascular outcomes in type 2 diabetes. N Engl J Med. 2015;373(3):232-42.

11. Gilbert RE, Mann JF, Hanefeld M, et al. Basal insulin glargine and microvascular outcomes in dysglycaemic individuals: results of the Outcome Reduction with an Initial Glargine Intervention (ORIGIN) trial. Diabetologia. 2014;57(7):1325-31.

12. Scandinavian Simvastatin Survival Study Group. Randomised trial of cholesterol lowering in 4444 patients with coronary heart disease: the Scandinavian Simvastatin Survival Study (4S). Lancet. 2004;344(8934):1383-9.

13. Yusuf S, Sleight P, Pogue J, Bosch J, Davies R, $\begin{array}{llll}\text { Dagenais G. } \quad \text { Effects of an } & \text { of }\end{array}$ angiotensin-converting-enzyme inhibitor, ramipril, on cardiovascular events in high-risk patients. The Heart Outcomes Prevention Evaluation Study Investigators. New Engl J Med. 2000;342(3):145-53.

14. Kalra S, Gupta Y, Baruah M. Can cardiac autonomic neuropathy be a predictor of cardiovascular outcomes in diabetes? Indian J Endocrinol Metab. 2015;19(2):196-7.

15. Inzucchi SE, Zinman B, Wanner C, et al. SGLT-2 inhibitors and cardiovascular risk: proposed pathways and review of ongoing outcome trials. Diab Vasc Dis Res. 2015;12(2):90-100.

16. Kalra S. Sodium glucose co-transporter-2 (SGLT2) inhibitors: a review of their basic and clinical pharmacology. Diabetes Ther. 2014;5(2):355-66.

17. Kalra S, Baruah MP, Sahay R. Medication counselling with sodium glucose transporter 2 inhibitor therapy. Indian $\mathrm{J}$ Endocrinol Metab. 2014;18(5):597-9. 